Ethik Med $2004 \cdot 16: 70$ DOI 10.1007/s00481-004-0282-5

Online publiziert: 30 . Januar 2004

(c) Springer-Verlag 2004
Hans-Konrat Wellmer

Bielefeld

\section{Kommentar I}

$D_{\text {e }}$ er hier geschilderte Fall ist in vielerlei Hinsicht beispielhaft. Ein alter Mann (Ist er mit 65 Jahren alt?) wird wegen einer typischen „Altersfraktur“ (pertrochantere Femurfraktur) nach einem typischen Minimaltrauma (Sturz an einer Bordsteinkante) hospitalisiert und unfallchirurgisch mit einer dynamischen Hüftschraube versorgt. Der intra- und postoperative Verlauf war unkompliziert.

Eine gute medizinische Untersuchung und Betreuung umfasst aber, wie hier geschehen, den ganzen Menschen. Die deutliche Untergewichtigkeit des Mannes wurde als auffälliges Symptom gewertet und dementsprechend weiter abgeklärt. Die Untersuchungsschritte Sonographie und Endoskopie schlossen sich folgerichtig an; dies setzte ja in jedem Einzelschritt die vorherige Einwilligung des Mannes voraus. An dieser Stelle möchte ich erwähnen, dass sich keinerlei Hinweise auf das soziale Umfeld und familiäre Bindungen finden. Auch Angaben über Laborwerte fehlen, so dass es schwer fällt, sich ein Gesamtbild von dieser Patientenpersönlichkeit $\mathrm{zu}$ machen.

Falls seine Einwilligung zur Koloskopie vorlag, war er über mögliche Komplikationen, wie Blutung oder Perforation, aufgeklärt, die eine Notfalloperation erfordern würden.

Die gute pflegerische Überwachung erkannte rechtzeitig die intraabdominelle Blutung durch Milzriss und führte $\mathrm{zu}$ der entscheidenden Situation, ob eine Notoperation ohne schriftliche Einwilligung, ja gegen den ausdrücklichen Willen des Patienten, erlaubt ist oder nicht. Diese Entscheidung zwischen Salus aegroti und Voluntas aegroti beschäftigt die Rechtssprechung ei- gentlich erst seit wenigen Jahrzehnten. Vorher herrschte die paternalistische Ansicht vor, der Arzt wisse am besten, was dem Patienten nottut. Seit der Einführung des Begriffes „Autonomie des Patienten“ ist die Rechtslage umgekehrt. Heute hat der Arzt zu beweisen, dass er den Patienten entsprechend aufgeklärt hat und dessen schriftliche Einwilligung vorliegt.

In diesem Fall ergriffen die Ärzte die Initiative und handelten gegen den Willen des Patienten, „der lieber sterben wollte“.

Die Fallgeschichte endet hier gut. Es bleiben aber Fragen offen. Ein Mensch will sterben, weil er so allein ist, nur den Hotelportier als Gesprächspartner hat. Er lebt auf durch die Freude seiner Mitpatienten über die geglückte „Rettung“. Hier zeigt sich übrigens die oftmals sehr positive Wirkung des Mehrbettzimmers auf den Heilungsprozess eines Patienten.

$\mathrm{Ob}$ die Entscheidung der Chirurgen auch mit der Tatsache zusammenhängt, dass es sich ja um eine Komplikation eines vorangegangenen Eingriffs handelt, kann nur vermutet werden. Eine ungeklärte Komplikation belastet den Operateur in aller Regel mehr als alle schicksalhaften Verläufe.

\section{Korrespondierender Autor}

Prof. Dr. med. Hans-Konrat Wellmer

Künnekestraße 5, 33647 Bielefeld

E-Mail: hkwellmer@aol.com

Interessenkonflikt: Der korrespondierende Autor versichert, dass keine Verbindungen mit einer Firma, deren Produkt in dem Artikel genannt ist, oder einer Firma, die ein Konkurrenzprodukt vertreibt, bestehen. 